\title{
Black Holes in the Light of Field Theory and Quantum Mechanics
}

\author{
Ahmad Tarhini, Khalil Abou-Saleh \\ Physics Department, Faculty of Sciences, Lebanese University, Nabatieh, Lebanon \\ Email: tarhini.ahmad@gmail.com, kabousaleh@ul.edu.lb
}

How to cite this paper: Tarhini, A. and Abou-Saleh, K. (2019) Black Holes in the Light of Field Theory and Quantum Mechanics. Journal of High Energy Physics, Gravitation and Cosmology, 5, 612-622. https://doi.org/10.4236/jhepgc.2019.53033

Received: April 5, 2019

Accepted: May 13, 2019

Published: May 16, 2019

Copyright ( 2019 by author(s) and Scientific Research Publishing Inc. This work is licensed under the Creative Commons Attribution International License (CC BY 4.0).

http://creativecommons.org/licenses/by/4.0/

\begin{abstract}
The black hole is a region in space where things may fall into it but nothing can come out. We present a study of the physics of a black hole using a quantum field theory frame based on the WZW model in a suitable mathematical frame. Based on the Schwarzschild metric, we show the different regions of our universe with the present singularities. Then we introduce the calculation of a black hole mass using the perturbation theory. We further discuss Hawking radiation and its quantum mechanical implications. At some limits, the space time can represent a black hole with a singularity hidden by the horizon.
\end{abstract}

\section{Keywords}

Black Holes, Field Theory, Schwarzschild Solution, Hawking Radiation

\section{Introduction}

One of the most interesting problems in string theory is the study of the solution of a black hole in two dimensions. So far, we have not been able to solve the many conceptual problems of 4-dimensional black holes. With the hope that string theory provides a natural framework for addressing these problems in a simplified context, Witten, in his articles [1] [2] [3], has proposed that exact conformal field theory that describes a black hole in space-time to two dimensions can be formulated as a $S L(2, \Re) / U(1)$ gauge of the Wess-Zumino-Witten (WZW) model.

WZW models are examples of nonlinear sigma models whose classical fields take values in non-flat target varieties [3]. For WZW models, the targets are Lie groups. We work in two dimensions CFT to simplify and we have two versions: Euclidian and Lorentzian, which are connected by a Wick rotation.

Consider an application $g: \Sigma \rightarrow M$ acting from a space $\Sigma$ called world- 
sheet space to a space $M$ called target space. If the metric of $M$ is non linear, then the model is named sigma model [4].

According to the choice of the Lie group $M$, one can build the Lagrangian theory, and if the choice is $M=S L(2, \Re) / U(1)$ we obtain a consistent theory that describes a black hole with a target space in two dimensions of space time. If the group is $\operatorname{SL}(2, C)$, then there is a complex Lagrangian.

The space world-sheet [5] describes the string that has a dimension, and when moving, it describes a two-dimensional surface. The theory is for bosonic strings (where there is an imaginary unstable particle of negative square mass called tachyon), the central charge is $c=2+\frac{6}{k+2} \quad(c=26$ in string theory) and $k$ is the algebra level of currents that can take values which are not integer because the representations are not unitary [5] [6].

The choice of $M=S L(2, \Re)$ [7] [8] gives an ungauged $S L(2, \Re)$ WZW action and one needs a gauge fixing to get a consistent and invariant theory so we divide by $U(1)$ [9] [10]. The possibility of choosing a gauge other than $U(1)$ help to obtain a black Euclidian or Lorentzian hole (which is obtained by an analytic continuation i.e. Wick rotation).

With this in mind, in the current context, starting from the above introduction, this paper is organized as follows: In Section 2, we introduce the WZW action in addition to mathematical group used and the corresponding metric. Then in Section 3, we explain the presence of the dilaton field and the comparison with the Liouville theory. In Section 4, we shall explore and discuss the analytical continuation of an Euclidean black hole. In Section 5, we will show the tachyon field and the singularity. Then in Section 6, we show the calculation of the black hole mass which is a consequence of time translation invariance. We present in Section 7 the Hawking radiation, and Section 8 is devoted to our conclusions.

\section{WZW Action}

The ungauged WZW action has the following form [3]:

$$
L(g)=\frac{k}{8 \pi} \int_{\Sigma} \sqrt{h} h^{i j} \operatorname{Tr}\left(g^{-1} \partial_{i} g g^{-1} \partial_{j} g\right)+i k \Gamma,
$$

with $\Sigma$ is a Riemanian surface of metric $h^{i j}$, and $\int \mathrm{d}^{2} x \sqrt{\operatorname{det} h^{i j}}$ to obtain an invariant form, and $g$ is the variable field of the model.

The term $i k \Gamma$ (Wess-Zumino term) is added for topological reasons and makes cancellations of anomalies. Without having to add it, we will have a massive theory at the quantum level that is non-conformal.

The group $S L(2, \Re)$ is a noncompact 3-dimensional group and has three generators so three scalar fields $g$. The metric of the form $(-,+,+)$ is undefined and we will have problems with negative magnitudes. The lagrangian (1) has a global symmetry $S L(2, \Re) \times S L(2, \Re)$ which corresponds to an application $g \rightarrow a g b^{-1}$ where $a, b$ belong to $S L(2, \Re)$. But to move to a local symmetry, 
it is necessary to fix the gauge by dividing by $U(1)$ of dimension 1 , then one obtain the two-dimensional metric $(+,+)$ which is completely Euclidian.

This gauge fixation is equivalent to kill a degree of freedom, thus killing a field $g$ of $S L(2, \mathfrak{R})$ where $g$ is in a specific frame (unitary gauge) [7] [11].

Introducing then an abelian gauged field $A$; As we know that $U(1) \simeq S O(2)$ (rotation in the plane) and in order to obtain a gauge invariance with $S L(2, \Re) / U(1)$, one can fix the gauge by choosing:

$$
g=\cosh r \cdot \mathbb{I}+\sinh r\left(\begin{array}{cc}
\cos \theta & \sin \theta \\
\sin \theta & -\cos \theta
\end{array}\right) .
$$

Since the lagrangian contains a quadratic part in A, we say that A is not dynamic or does not have a kinetic term and it does not appear in the equations of motion, and the term of $\mathrm{WZ}$ contains a total derivative then we can forget them in the treatment of integrals. The following action represents then the lagrangian of the sigma model and the metric of $S L(2, \mathfrak{R})$ :

$$
I(r, \theta)=\frac{k}{4 \pi} \int \mathrm{d}^{2} x \sqrt{h} h^{i j}\left(\partial_{i} r \partial_{j} r+\tanh ^{2} r \partial_{i} \theta \partial_{j} \theta\right) .
$$

For large $r,(r \rightarrow \infty), \tanh r \rightarrow 1$ (Euclidian metric $(+,+))$, then the metric of the target space of the theory is $\mathrm{d} s^{2}=\frac{k}{2} \mathrm{~d} \sigma^{2}$ with

$$
\mathrm{d} \sigma^{2}=(\mathrm{d} r)^{2}+\tanh ^{2} r(\mathrm{~d} \theta)^{2} .
$$

The metric has the shape of a semi-infinite cigar (see Figure 1); it is asymptotic when $r \rightarrow \infty$ to $\mathfrak{R} \times S^{1}$. This space-time is a good candidate to represent a black hole [2] [3].

\section{Dilaton Field and Curvature}

Taking into account the quadratic term of the abelian field is similar to adding a coupling term to the action that represents the interaction between the expansion field $\Phi$ (function of the target space) and the curvature $R^{(2)}$ of the metric of the world-sheet space $h$ [12] [13] [14].

$$
I(r, \theta)=\frac{k}{4 \pi} \int \mathrm{d}^{2} x \sqrt{h} h^{i j}\left(\partial_{i} r \partial_{j} r+\tanh ^{2} r \partial_{i} \theta \partial_{j} \theta\right)-\frac{1}{8 \pi} \int \mathrm{d}^{2} x \sqrt{h} \Phi(r, \theta) R^{(2)},
$$

With this lagrangian, we will have a conformal invariance, then the $\beta$ function of the coupling vanishes and we obtain the equation of motion $R_{a b}=D_{a} D_{b} \Phi$ where $R_{a b}$ is the Ricci tensor of the target space which has a solution:

$$
\Phi=2 \ln (\cosh r)+\text { constant }
$$

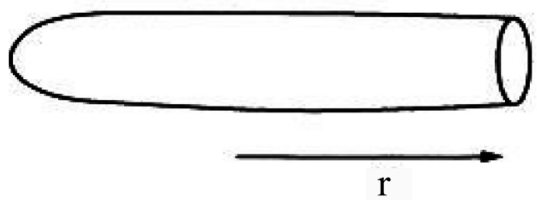

Figure 1. A semi infinite cigar. 
This constant plays an important role in determining the mass of the black hole as we will see later. We notice that this constant doesn't affect the string coupling $g_{S}$ which is proportional to $\mathrm{e}^{-\langle\Phi\rangle}$ since $\langle$ constante $\rangle=0$ $\left(\mathrm{e}^{-\langle\Phi\rangle+c t e}=\mathrm{e}^{-\langle\Phi\rangle}\right)$.

When $r \rightarrow \infty$ (asymptotic region at the end of the cigar) then $\Phi \rightarrow \infty$ and $g_{S} \rightarrow 0$. Therefore, the coupling is weak and one can use the perturbation theory.

We deduce that we can calculate the central charge in the asymptotic region and generalize it everywhere. Note that $c=2+\frac{6}{k}$ is a little different from initial $c$ because we made an incomplete calculation in the gauge fixing but the asymptotic behavior of the dilaton field remains the same because in this minimal coupling $1 / k \sim 1 / k^{\prime}=1 / k-2$.

We can make a comparison with the Liouville theory [15] which is with a scalar field coupled with gravity and has two coordinates $\varphi, \theta$ and we find that $\varphi$ plays the same role as $r$ with the same conditions at limits. This leads us to replace the standard Liouville theory with an Euclidean black hole (i.e. no temporal component). It is always necessary to choose an action with counter-terms so that we will have an invariant conformal theory, and it is necessary to specify the infinitesimal variations of the transformations of the fields with a good gauge fixing.

According to the light cone, the space-like and time-like regions are disconnected and we cannot go from one to the other by a Lorentz transformation but we can use a conformal transformation to eliminate a coordinate of time or space (either $X^{0}=0$ or $X^{1}=0$ ). And so we deduce a theory of critical strings at $D$ dimensions from another non-critical at $(D-1)$ dimensions.

Consider the transformation of Liouville for an Euclidean black hole where a degree of freedom is gauged away:

$$
\delta r=\epsilon \frac{1}{2 k^{\prime}} \tanh r, \quad \delta \theta=0 .
$$

if $r$ tends to infinity then one can eliminate it and we obtain a single degree of freedom which is the scalar field $\theta$ to a single dimension.

\section{Analytical Continuation and Lorentz Signature of a Black Hole}

To discuss the Lorentzian black hole, the analytic continuation of the Euclidean, we make a Wick rotation $\theta=i t$ then the metric will be:

$$
\mathrm{d} \sigma^{2}=(\mathrm{d} r)^{2}-\tanh ^{2} r(\mathrm{~d} t)^{2},
$$

where $r$ is Liouville coordinate interpreted as spatial coordinate, and $\theta$ is for time. In this metric, we have a singularity due to the choice of coordinates and not a physical singularity because the scalar curvature $R=\frac{4}{\cosh ^{2} r}$ is regular for $r=0$. 
Similar to the case of the Schwarzschild metric in four dimensions ${ }^{1}$, we can use a suitable change of variables (Kruskal coordinates): $r^{\prime}=r+\ln \left(1-\mathrm{e}^{-2 r}\right)$, we have

$$
\mathrm{d} \sigma^{2}=\tanh ^{2} r\left[\left(\mathrm{~d} r^{\prime}\right)^{2}-(\mathrm{d} t)^{2}\right]
$$

and with

$$
2 u=-\mathrm{e}^{r^{\prime}-t}, 2 v=\mathrm{e}^{r^{\prime}+t} \Rightarrow 1-u v=\cosh ^{2} r, \sinh ^{2} r=-u v,
$$

we get

$$
\mathrm{d} \sigma^{2}=-\frac{\mathrm{d} u \mathrm{~d} v}{1-u v}
$$

We obtain the analytically continued black hole space time represented in Figure 2.

Clearly, region I is our asymptotically flat half-space. The physical singularity is at $u v=1$ because the curvature blows up:

$$
R=\frac{4}{\cosh ^{2} r}=\frac{4}{1-u v} \rightarrow+\infty .
$$

Region II: Future branch which is the black hole singularity, from which no signal can cross the horizon to an observer in region I.

Region III: Past branch which is the white hole-the time reversal of black holes. This region contains naked singularity i.e. a gravitational singularity without an event horizon.

Region IV: Is another asymptotically flat region where no signal can propagate from this region to region I and vice versa. Now, for $u v>1$, one can find two additional asymptotically flat spaces regions V and VI. It should be noted that the singularity at $u v=1$ appears at the end of the spatial world to an observer in region $\mathrm{V}$ or $\mathrm{VI}$ and it is a naked singularity.

One can verify that regions $\mathrm{V}$ and VI are space-times with negative mass squared. Indeed, this is due to the presence of unstable sub-atomic particle called Tachyon. It moves faster than the speed of light, posses an imaginary mass and vibrates in open strings in the string theory.

Such regions with negative mass singularity appear in the four dimensional Schwarzschild solution if one continues past the singularity. One might think that the singularity of the black hole is a result of bad gauge choice. However we can argue that this is not the right interpretation.

Indeed given the initial data in region I one cannot without solving the problem of free will predict whether a black hole will form because there is time like loops that can lead to possibility of killing one's own remote ancestors. To obtain a consistent conformal theory, it is necessary to work in regions that do not contain singularities, i.e. Orbifold $/ Z_{2}{ }^{2}$. Then the remaining regions are (I, IV).

'Schwarzschild metric: $\mathrm{d} s^{2}=-\left(1-\frac{2 G M}{r}\right) \mathrm{d} t^{2}+\left(1-\frac{2 G M}{r}\right)^{-1} \mathrm{~d} r^{2}+r^{2} \mathrm{~d} \Omega^{2}$.

${ }^{2}$ Orbifold: region with singularity. We have 2 strings: open string $(1 / r)$ and closed string (circle $r$ ) which are dual. The compression of the circle gives a segment and the singularities are at the end. 


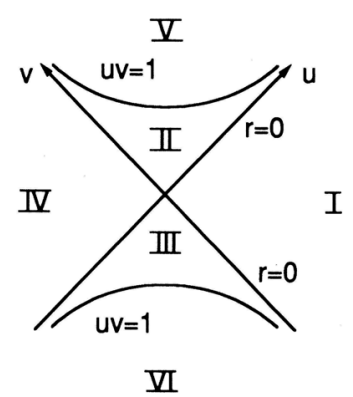

Figure 2. The analytically continued black hole space time.

In order to obtain the Lorentzian form of a black hole with an analytical continuation, one can use a conformal theory of the fields by gauging different subgroups of $S L(2, \Re)$.

$$
g=\left(\begin{array}{cc}
a & u \\
-v & b
\end{array}\right), \quad a b+u v=1
$$

The gauge invariance are:

$$
\begin{aligned}
& \delta a=2 \epsilon a \\
& \delta b=-2 \epsilon b \\
& \delta u=\delta v=0 \\
& \delta A_{i}=-\partial_{i} \epsilon
\end{aligned}
$$

Then, one can obtain a gauged WZW action after coupling with an auxiliary field $A$.

$$
\begin{aligned}
L= & -\frac{k}{4 \pi} \int \mathrm{d}^{2} z\left(\partial_{z} u \partial_{\bar{z}} v+\partial_{\bar{z}} u \partial_{z} v+\partial_{z} a \partial_{\bar{z}} b+\partial_{\bar{z}} a \partial_{z} b\right) \\
& +\frac{k}{2 \pi} \int \mathrm{d}^{2} z\left[A_{\bar{z}}\left(b \partial_{z} a-a \partial_{z} b+u \partial_{z} v-v \partial_{\bar{z}} u\right)\right. \\
& +A_{z}\left(b \partial_{\bar{z}} a-a \partial_{\bar{z}} b-u \partial_{\bar{z}} v+v \partial_{\bar{z}} u\right) \\
& \left.+A_{z} A_{\bar{z}}(4-4 u v)+\ln a\left(\partial_{\bar{z}} u \partial_{\bar{z}} v-\partial_{\bar{z}} u \partial_{\bar{z}} v\right)\right]
\end{aligned}
$$

where $a$ and $b$ are family of group element of $\operatorname{SL}(2, \Re)$ manifold. A gauge fixing $a=b$ is equivalent to kill one degree of freedom. In the region where $1-u v>0, a b+u v=1 \Rightarrow a>0, b>0$ or $a<0, b<0$. Therefore we obtain two copies of regions I, IV. If $a=-b$ then $1-u v<0$ and we obtain two copies of regions $\mathrm{V}$, VI.

We can also see this result in a mathematical way by reasoning on $\operatorname{SL}(2, \Re)$ which has 2 copies and its universal covering contain an infinity of copies. To explain this idea, let's take an example: The metric in this case is Lorentzian (and not Euclidean), from a mathematical point of view, $S L(2, \Re)$ is not divided by $U(1)$ which is a compact group but by $S O(1,1)$ which is non-compact to 1 dimension so it remains the Lorentzian metric only.

To fix the problem of simple connectivity, one should use the universal covering:

$$
\text { (universal cover }) /(\text { fundamental group }) \simeq(\text { space itself })
$$


There is an analogy between $S L(2, \Re)$ and $S U(2) / Z_{2} \simeq S O(3) . S U(2)$ has 2 copies (spinor representation) and $S O(3)$ has an infinity of copies. $S U(2)$ is simply connected but $S O(3)$ is not, then there is an algebraic object that measures the default of $S O(3)$ to be simply connected which is $\pi_{1}(S O(3)) \simeq Z_{2}$.

\section{Tachyon Field and Singularity}

Recall that the study of a black hole is equivalent to the study of the $\sigma$-Model metric [4], then this analogy with the groups gives us a lot of information and symmetries in the black hole. In this gauge $a=b$ and after the elimination of the auxiliary field $A$, we will have this action:

$$
L=-\frac{k}{4 \pi} \int \mathrm{d}^{2} x \sqrt{h} h^{i j} \frac{\partial_{i} u \partial_{j} v}{1-u v} .
$$

The singularity at $u v=1$ i.e. $r \rightarrow \infty$ treats the asymptotic region where the perturbation theory can be applied in $1 / k$. We consider for this the tachyon because it is unstable and disappears but it gives physical results. Let us consider the propagation of a small tachyon disturbance in the black hole background. Because of the underlying $S L(2, \Re) / U(1)$, it may be possible to solve exactly for the tachyon vertex operators, but we will simply consider perturbation theory in $\frac{1}{k}$. The Dilaton is a particle of a scalar field $\phi$, follows the KleinGordon equation and couples with gravity. The exponential of its vacuum expectation value determines the coupling constant: $g=\mathrm{e}^{\langle\phi\rangle}$. Therefore the coupling constant is a dynamical variable in string theory, unlike the case of quantum field theory where it is constant. The tachyon field $T(u, v)$, to lowest order, is governed by an effective action in space-time:

$$
L(T)=\int \mathrm{d}^{2} X \sqrt{g} \mathrm{e}^{\phi}\left[g^{i j} \partial_{i} T \partial_{j} T-8 T^{2}\right] .
$$

In the black hole space time, this action is:

$$
L(T)=\int \mathrm{d} u \mathrm{~d} v\left[(1-u v) \partial_{u} T \partial_{v} T-\frac{16}{k^{\prime}} T^{2}\right] .
$$

The tachyon field equations are therefore:

$$
\partial_{u}\left((1-u v) \partial_{v} T\right)+\partial_{v}\left((1-u v) \partial_{u} T\right)+\frac{16}{k^{\prime}} T=0 .
$$

One can look for ansatz solution in the form $T(u, v)=\left(\frac{u}{v}\right)^{\gamma} f(u, v)$ using the symmetry of space-time under: $\left(u \rightarrow \mathrm{e}^{t} u, v \rightarrow \mathrm{e}^{-t} v\right)$. We then reach to a second order differential equation which has two linearly independent solutions:

A regular solution near $x=1-u v=0$, that can be expanded in power series, $f(x)=1+\sum_{n=1}^{\infty} a_{n} x^{n}$.

A logarithmic solution singularity, $f(x)=\ln x+O(x \ln x)$. The physical problems of the black hole are not solved, despite the fact that the conformal theory is non-singular at $u v=1$. 


\section{Black Hole Mass}

About the mass of a black hole, we know that the metric in general can be written as $\mathrm{d} s^{2}=g_{t t}(r) \mathrm{d} t^{2}+$ spherical term. If we take $g_{t t}(r)=0$ then we can find the horizon, and if we apply Taylor expansion of $g_{t t}(r)$ then the term of order $(1 / r)$ contains a constant coefficient which is the mass that is called ADM mass.

Let us write the effective action, to the lowest order in world-sheet perturbation theory, of the graviton-dilaton system:

$$
L(T)=\int \mathrm{d}^{2} X \sqrt{g} \mathrm{e}^{\phi}\left[R+g^{i j} \partial_{i} \Phi \partial_{j} \Phi+\frac{8}{k^{\prime}}\right],
$$

we deduce the equation of motion for the gravitational field:

$$
Q_{i l}=-D_{i} D_{l} \Phi+g_{i l}\left[D_{k} D^{k} \Phi+\frac{1}{2} D_{k} \Phi D^{k} \Phi-\frac{4}{k^{\prime}}\right]=0,
$$

and the equation of motion for the dilaton field:

$$
2 D_{k} D^{k} \Phi+D_{k} \Phi D^{k} \Phi-R-\frac{8}{k^{\prime}}=0 .
$$

These equations have a flat solution (standard Liouville solution) in a spacetime of coordinates $\rho$ and $\tau$. This solution is also an asymptotic solution of a black hole (of coordinates $r$ and $t$ ) with a simple change of variables.

$$
\mathrm{d} s^{2}=\mathrm{d} \rho^{2}-\mathrm{d} \tau^{2}, \quad \Phi=\rho \sqrt{\frac{8}{k^{\prime}}} .
$$

This solution is invariant under time translations and hence a conserved energy in the fluctuation about it, so the mass is conserved. Therefore we can study the asymptotic region of a black hole to know its mass.

After the calculation of the asymptotically conserved current $S_{i}$, the conserved charge $S_{0}$ is calculated by making a perturbation of the solution and the metric to have an analogy between

$$
\Phi=\rho \sqrt{\frac{8}{k^{\prime}}}+\varphi
$$

where $\varphi$ is zero at infinity and

$$
\Phi=2 \ln (\cosh r)+a .
$$

Consider the solution (23) that is asymptotic to the flat solution space with

$$
g_{i j}=\eta_{i j}+h_{i j} .
$$

Note that $\varphi$ and $h$ vanish for $\rho \rightarrow \infty$, and $\eta$ is the flat space metric corresponding to $\mathrm{d} \rho^{2}-\mathrm{d} \tau^{2}$. Let $Q_{i j}=q_{i j}+$ terms of higher orders; where $q_{i j}$ are the linear terms of $Q_{i j}$. The quantity $S_{i}=q_{i j} v^{j}$ is the asymptotically conserved current, where $v^{j}$ the time translation generator. The corresponding conserved charge density $S_{0}$ is always a total divergence and as a result the conserved charge can be measured as a surface term at $\rho \rightarrow+\infty$. After long straight forward calculation of $S_{0}$, we then get the mass $\mathrm{M}$ of the black hole at $\rho \rightarrow+\infty$ : 


$$
M=\sqrt{\frac{2}{k^{\prime}}} \mathrm{e}^{a} .
$$

We see that the significance of the possibility of adding a constant to the dilaton field will determine the mass of the black hole. It is important to notice that adding a constant $\Phi$ does not change the physical state at $\rho \rightarrow+\infty$ since we can always absorb this constant in the translation of $\rho$. This is why the black hole with $\Phi=2 \ln (\cosh r)+a$ can be regarded as a family of objects of variable mass inserted in a fixed space-time background. At the horizon, the value of the dilaton field is:

$$
\Phi(r=0)=a=\ln \left(M \sqrt{\frac{k^{\prime}}{2}}\right)
$$

\section{Hawking Radiation}

Stephen Hawking states that black holes have finite non-zero temperature obtained by the inverse of the circle radius at infinity in a solution of an Euclidean black hole [16]-[21]. According to the quantum mechanical uncertainty principle, black holes should create and emit particles. The emission process is known as the Hawking radiation, reduces the mass of the black hole and leads therefore to the black hole evaporation. According to (27), the mass loss means that the value of the dilaton field on the horizon will diminish and thus the string coupling constant will get stronger there. For massless black hole, the physics approaches more and more the idealized flat space solution. Because $M \rightarrow 0$ means that $a \rightarrow-\infty$, and consequently from (26) $\Phi \rightarrow \rho \sqrt{\frac{8}{k^{\prime}}}$. Thus this flat space solution would appear to be the end point of the black-hole evaporation which produce two copies of the standard space time [22]. The flat solution (22) is the solution with the greatest symmetry and is described asymptotically i.e. the space time is the end point of Hawking radiation. This space time can represent a black hole with a singularity hidden by the horizon.

Consider a perturbation of this flat space by sending a particle of energy $\epsilon$. To make an analogy with a black hole, it is necessary that the particle exceeds what is called Schwarzschild radius in 4 dimensions and which will be, in our case, the coordinate $\rho$ :

$$
\rho(\epsilon)=\sqrt{\frac{k^{\prime}}{8}} \ln \left(\epsilon \sqrt{\frac{8}{k^{\prime}}}\right) .
$$

Either the particle will be reflected before reaching its Schwarzschild radius. Or if it reaches its Schwarzschild radius, a horizon will appear to form but presumably the energy $\epsilon$ involved will be re-emitted as some form of Hawking radiation. Consequently, what we expect to see is a quantum-mechanical $S$ matrix, with particles coming in and ultimately being ejected in one form or another.

To determine whether the $S$ matrix is a reflection or radiation of particles, we 
will introduce the cosmological constant: A tachyon field with $T(\rho, \tau)=\mu \mathrm{e}^{-\sqrt{8} \rho}$ which can be the perturbation added to the flat solution (22). Introducing this field in the $\Phi$ of the flat space, will affect the suppressing string propagation into the region $\rho \rightarrow-\infty$ where the string coupling become strong.

So the possibility of changing the radius of the circle at infinity i.e. the temperature of the black hole is equivalent to change the cosmological constant because, as we have seen, it is the perturbation in the solution of an Euclidean black hole.

In the light of this context, we consider the recent improvement to Hawkings evaporation theory, that is the so called Bohr-like approach to black hole quantum physics [23] [24] [25]. It has been shown that, in the semi-classical approximation, the Schwarzschild black hole is the gravitational analogous of Bohr hydrogen atom. This approach is important not only in the context of black holes in the light of quantum mechanics, but also because it presents a strong, independent solution to the black hole information paradox.

\section{Conclusion}

In the present work, we studied the solution of a black hole in a field theory frame based on the concepts of general relativity in a gauged WZW model. We have reviewed the WZW action, the different region of a black hole space time and the gauge fixing in a suitable mathematical frame. The black hole does not provide us by information from this point of view. However, from quantum mechanics, Hawking radiation tells us that black holes emit radiations i.e. give information. So we cannot put general relativity and quantum mechanics in the same frame.

\section{Conflicts of Interest}

The authors declare no conflicts of interest regarding the publication of this paper.

\section{References}

[1] Witten, E. (1991) Strings: StonyBrook.

[2] Witten, E. In: Mansouri, F. and Scanio, J.J., Eds., Quantum Gravity and Beyond, 126-142.

[3] Witten, E. (1991) String Theory and Black Holes. Physical Review D, 44, 314. https://doi.org/10.1103/PhysRevD.44.314

[4] Ohmori, K., Seiberg, N. and Shao, S.H. (2019) Sigma Models on Flags. SciPost Physics, 6, 017. https://doi.org/10.21468/SciPostPhys.6.2.017

[5] Eberhardt, L., Gaberdiel, M.R. and Gopakumar, R. (2018) The Worldsheet Dual of the Symmetric Product CFT.

[6] Seiberg, N. and Witten, E. (1999) The D1/D5 System and Singular CFT. JHEP, 9904, 017. https://doi.org/10.1088/1126-6708/1999/04/017

[7] Eghbali, A. (2019) Exact Conformal Field Theories from Mutually T-Dualizable $\sigma$-Models. Physical Review D, 99, Article ID: 026001. https://doi.org/10.1103/PhysRevD.99.026001 
[8] Kiritsis, E. (1993) Exact Duality Symmetries in CFT and String Theory. Nuclear Physics B, 405, 109-142. https://doi.org/10.1016/0550-3213(93)90428-R

[9] Alekseev, A., Klimicik, C. and Tseytlin, A. (1996) Quantum Poisson-Lie T-Duality and WZNW Model. Nuclear Physics B, 458, 430-444. https://doi.org/10.1016/0550-3213(95)00575-7

[10] Lledo, M.A. and Varadarajan, V.S. (1998) Su (2) Poisson-Lie T Duality. Letters in Mathematical Physics, 45, 247-257. https://doi.org/10.1023/A:1007498803198

[11] Sfetsos, K. (1998) Poisson-Lie T-Duality beyond the Classical Level and the Renormalization Group. Physics Letters B, 432, 365-375.

https://doi.org/10.1016/S0370-2693(98)00666-2

[12] Soroushfar, S., Saffari, R. and Kamvar, N. (2016) Thermodynamic Geometry of Black Holes in $\mathrm{f}(\mathrm{R})$ Gravity. The European Physical Journal C, 76, 476. https://doi.org/10.1140/epjc/s10052-016-4311-6

[13] Saffari, R. and Rahvar, S. (2008) f(R) Gravity: From the Pioneer Anomaly to Cosmic Acceleration. Physical Review D, 77, Article ID: 104028. https://doi.org/10.1103/PhysRevD.77.104028

[14] Soroushfar, S., Saffari, R., Kunz, J. and Lammerzahl, C. (2015) Analytical Solutions of the Geodesic Equation in the Spacetime of a Black Hole in $\mathrm{f}(\mathrm{R})$ Gravity. Physical Review D, 92, Article ID: 044010. https://doi.org/10.1103/PhysRevD.92.044010

[15] Polyakov, A.M. (1981) Quantum Geometry of Bosonic Strings. Physics Letters B, 103, 207-210. https://doi.org/10.1016/0370-2693(81)90743-7

[16] Hawking, S.W. (1975) Particle Creation by Black Holes. Communications in Mathematical Physics, 43, 199-220. https://doi.org/10.1007/BF02345020

[17] Wald, R.M. (1994) Quantum Field Theory in Curved Space-Time and Black Hole Thermodynamics. University of Chicago Press, Chicago.

[18] Ghosh, A. (2019) Hawking Radiation-Revisited.

[19] Brout, R., Massar, S., Parentani, R. and Spindel, P. (1995) Hawking Radiation without Trans-Planckian Frequencies. Physical Review D, 52, 4559. https://doi.org/10.1103/PhysRevD.52.4559

[20] Visser, M. (1998) Acoustic Black Holes: Horizons, Ergospheres, and Hawking Radiation. Classical and Quantum Gravity, 15, 1767. https://doi.org/10.1088/0264-9381/15/6/024

[21] Singleton, D. and Wilburn, S. (2011) Hawking Radiation, Unruh Radiation and the Equivalence Principle. Physical Review Letters, 107, Article ID: 081102. https://doi.org/10.1103/PhysRevLett.107.081102

[22] Katsuragawa, T. and Nojiri, S. (2015) Stability and Antievaporation of the Schwarzschild-de Sitter Black Holes in Bigravity. Physical Review D, 91, Article ID: 084001. https://doi.org/10.1103/PhysRevD.91.084001

[23] Corda, C. (2015) Precise Model of Hawking Radiation from the Tunnelling Mechanism. Classical and Quantum Gravity, 32, Article ID: 195007. https://doi.org/10.1088/0264-9381/32/19/195007

[24] Corda, C. (2015) Time Dependent Schrödinger Equation for Black Hole Evaporation: No Information Loss. Annals of Physics, 353, 71-82. https://doi.org/10.1016/j.aop.2014.11.002

[25] Corda, C. (2015) Quasi-Normal Modes: The "Electrons" of Black Holes as "Gravitational Atoms"? Implications for the Black Hole Information Puzzle. Advances in High Energy Physics, 2015, Article ID: 867601. https://doi.org/10.1155/2015/867601 